\title{
Essential Pain Pharmacology (The Prescriber's Guide)
}

\author{
Howard S. Smith, Marco Pappagallo, Consultant Editor: Stephen M. Stahl. Cambridge \\ University Press, 561 Pages, ISBN 978-0-521-75910-6
}

\author{
Reza Tabrizchi, PhD
}

Received: 5 April 2013/Accepted: 11 April 2013/Published online: 18 April 2013

(C) Canadian Anesthesiologists' Society 2013

The editors present a comprehensive text that deals with pertinent information regarding the numerous substances available for pain management. Practitioners are also provided guidance on options available for treating populations that do not respond well to standard pharmacotherapy directed at alleviation of pain.

The text is presented as a straightforward menu detailing therapeutics, adverse effects, and dosing, as well as use of drugs in special populations. As an added feature, each section includes a concise perspective on the pharmacological actions of every substance. Here, the contributors endeavour to describe potential advantages/disadvantages, primary targets, and nuggets of information on how to deliver effective pain management and reduce adverse effects.

The authors make use of visual cues in this text to simplify the flow of information for the reader. For example, a section that originates with a discussion in therapeutics leads to particulars regarding cataloguing class, use, mechanism(s) of action, and responders/nonresponders and is capped with the best way to augment the actions of a substance as an analgesic. The text clearly defines the relevant issues relating to pharmacodynamics and, where possible, incorporates details regarding the pharmacokinetics of the formulation used for alleviation and management of pain. The authors also endeavour to provide solid information on the use of analgesics in special populations, i.e., those with renal, hepatic, and cardiac impairment as well as in the elderly, children, pregnancy, and nursing mothers. Where available, this amount of

R. Tabrizchi, PhD ( $($ )

Division of BioMedical Sciences, Memorial University,

St. John's, NL, Canada

e-mail: rtabrizc@mun.ca detail regarding pain medication should be particularly helpful for defining a therapeutic regimen for patients who experience or will experience nociceptive stimuli during a procedure or event.

Dose and route of delivery are succinctly defined and the dialogue leads to dosing tips and overdose. The information in this section was likely difficult to compile considering the compact nature of this reference text. There is a vast volume of data to deal with as well as uncertainties concerning some of the noted interactions among various pharmaceutical agents. Furthermore, the sensitivity of individual patients varies as a result of their unique demographics. Nevertheless, the authors have made a very credible effort to address this subject. There is a clear need to strike a balance, and compromises must be made when selecting and compiling data on substances used in the management of pain. The contributors of this reference book have successfully achieved a balance between imparting condensed information and incorporating as many critical facts as possible for each substance documented in the text. In essence, this text offers a reasonable volume of pharmacological and therapeutic information on a wide variety of substances that are useful in the management of pain.

A section of the text is devoted to nutraceuticals and medical food preparations for the treatment of chronic pain. Many of these substances have antioxidant properties that seem to cover the pharmacological basis for management of pain. There is very modest information on these groups of substances for special populations, which may be linked directly to the absence of substantial controlled clinical trials. Nevertheless, in certain conditions, these nutraceuticals and medical food preparations appear to be reasonable adjuncts to medicinal drugs used for the management of chronic pain. 
It is also recognized that pain is a very subjective experience and can vary greatly among individuals within a population. The asymmetrical nature of pain sensation among individuals, coupled with variable pharmacokinetics and pharmacodynamics of the molecules utilized in the management of this condition, can sometimes make strategies for analgesia in a diverse population quite challenging. A reference text such as this should provide a broad array of compounds that would be helpful to physicians who are attempting to alleviate pain in patients. The editors of this text have largely achieved this task. For example, the pyramid schematic describing first, second, third, and fourth-line analgesics is an effective and useful visual tool to determine the drug or drug combinations that would be appropriate and beneficial in the management of pain.

In addition, the pharmacological description for each substance has been annotated under various pertinent subheadings that guide the reader to useful and helpful therapeutic information. The text also includes suggested additional reading material that is fairly up to date and would be of value for the reader.

Furthermore, the method of indexing is particularly helpful, as substances utilized for management of pain are catalogued under a number of useful headings, i.e., by name, use, and class. This makes the task of searching for a substance for alleviation of pain easier and more efficient.

Overall, this is a helpful compact text with a reasonable volume of information on a large body of medicinal drugs, nutraceuticals, and medical food preparations for the management of pain.

Conflicts of interest None declared. 